\title{
Fluorescent fullerene nanoparticle-based lateral flow immunochromatographic assay for rapid quantitative detection of C-reactive protein
}

\author{
Kyung Mi Park' ${ }^{1}$ Da Jung Chung ${ }^{1}$, Mijin Choi ${ }^{1}$, Taejoon Kang ${ }^{2,3^{*}}$ (1) and Jinyoung Jeong ${ }^{3,4^{*}}$
}

\begin{abstract}
A fluorescent fullerene nanoparticle (NP)-based lateral flow immunochromatographic assay (LFIA) was developed for the rapid and quantitative detection of C-reactive protein (CRP) in serum. The polyclonal CRP-antibody-conjugated fullerene NPs were simply prepared by 1-ethyl-3-(3-dimethyllaminopropyl)-carbodiimide hydrochloride coupling after carboxylation of fluorescent fullerene NPs. By applying the CRP-antibody-conjugated fullerene NPs to a lateral flow test strip, quantitative analysis of CRP in serum was possible at a concentration range of $0.1-10 \mathrm{ng} / \mathrm{ml}$ within $15 \mathrm{~min}$. We anticipate that this novel fluorescent fullerene NP-based LFIA can be useful for the rapid and accurate sensing of biological and chemical species, contributing to the disease diagnosis and prognosis, environmental monitoring, and food safety.
\end{abstract}

Keywords: Fullerene, Nanoparticle, C-reactive protein, Lateral flow immunochromatographic assay, Fluorescence

\section{Introduction}

The lateral flow immunochromatographic assay (LFIA) is a common technique for the detection of such diverse analytes as hormones, disease-related biomarkers, and toxins in the clinical, environmental, and food industry fields, because of its simplicity and rapidity [1-8]. As a standard reporting material in LFIA, colloidal gold (CG) has been widely used for colorimetric detection due to its visibility. However, the CG-based LFIA often suffers from limitations such as lack of sensitivity and the ability to provide only qualitative/semi-quantitative analysis. To overcome the drawbacks of CG-based LFIA, various materials have been developed as reporters, including fluorescent microspheres (FMs), quantum dots (QDs), up-conversion nanoparticles (UCNPs), carbon nanoparticles (CNPs), and platinum nanoparticles (PtNPs) [9-13]. Although these reporter materials have enabled

\footnotetext{
*Correspondence: kangtaejoon@kribb.re.kr; jyjeong@kribb.re.kr

${ }^{3}$ Department of Nanobiotechnology, KRIBB School of Biotechnology, University of Science \& Technology, Daejeon 34113, Republic of Korea Full list of author information is available at the end of the article
}

sensitive and quantitative analyses of molecules even at low analyte concentrations, challenges remain in terms of material preparation, functionalization of the materials for efficient conjugation of the target molecules, and optimization of sensing conditions on a lateral flow assay. Previously, we developed a novel fluorescent fullerene material, tetraethylene glycol-conjugated fullerene nanoparticles $\left(\mathrm{C}_{60}\right.$-TEG), that was prepared via a simple procedure involving lithium hydroxide as a catalyst at room temperature $[14,15]$. These fluorescent fullerene nanoparticles (NPs) are easy to prepare compared to other inorganic materials, i.e., QDs and UCNPs, that require large amounts of surfactants, complex purification steps, and harsh conditions such as high temperatures for synthesis. Furthermore, the fullerene NPs can provide distinct and controllable fluorescent signals. These unique properties of $\mathrm{C}_{60}$-TEG prompted us to employ them for LFIA.

Herein, we report a new fluorescent probe $\left(\mathrm{C}_{60}\right.$-TEG)-based LFIA, for the highly sensitive, rapid, and quantitative analysis of $\mathrm{C}$-reactive protein (CRP) in serum. CRP is known as an acute-phase plasma 
protein that is a non-specific but sensitive inflammation marker, especially in the case of bacterial infection. It is also known as a potential indicator of cardiovascular disease, e.g., coronary heart disease, ischemic stroke, and acute myocardial infarction [16-18]. Because the measurement of low concentrations of CRP is critical for early diagnosis of inflammation and cardiovascular disease, many researchers have attempted to develop a highly sensitive CRP-detectable LFIA [19-21]. For example, Swanson et al. recently reported a CRP detection limit of $10 \mathrm{ng} / \mathrm{ml}$ using near-infrared dye-LFIA [22]. In this work, we demonstrated the quantitative analysis of CRP in the presence of serum with a wide dynamic range of $0.1-10 \mathrm{ng} / \mathrm{ml}$ by using the polyclonal anti-CRP-conjugated $\mathrm{C}_{60}$-TEG (pAb-CRP- $\mathrm{C}_{60}$-TEG) as a fluorescent probe. The $\mathrm{pAb}-\mathrm{CRP}-\mathrm{C}_{60}$-TEG was simply prepared by 1-ethyl-3-(3-dimethyllaminopropyl)-carbodiimide hydrochloride (EDC) coupling after carboxylation of fluorescent fullerene NPs. Since the developed $\mathrm{C}_{60}$-TEG-based LFIA achieves sufficiently high sensitivity and quantitative analysis of a target molecule, the $\mathrm{C}_{60}$-TEG-based LFIA can be used as an advanced fluorescent LFIA for disease diagnosis and prognosis, environmental monitoring, and food safety.

\section{Results and discussion}

2.1 Synthesis and characterization of $\mathrm{pAb}-\mathrm{CRP}-\mathrm{C}_{60}$-TEG

Figure 1 is synthetic procedure of pAb-CRP- $\mathrm{C}_{60}$-TEG for LFIA. Firstly, the $\mathrm{C}_{60}$-TEG was prepared by adding $\mathrm{LiOH}$ to a mixture of $\mathrm{C}_{60}$ and TEG. Then, the NPs were modified to expose a carboxylate group by the reaction with $\mathrm{SA}$ and DMAP. Next, the $\mathrm{C}_{60}$-TEG-COOH and $\mathrm{pAb}-\mathrm{CRP}$ were conjugated via EDC coupling (Fig. 1). This pAbCRP- $\mathrm{C}_{60}$-TEG preparation process is uncomplicated and easy to perform under ambient conditions, whereas other reporting materials, e.g., semiconducting QDs and UCNPs, are synthesized at high temperatures and in organic solvents. In addition, the $\mathrm{C}_{60}$-TEG-COOH does not need to be water-soluble intentionally because it is highly hydrophilic.

The absorption and fluorescence spectra of the $\mathrm{C}_{60}$-TEG-COOH are shown in Fig. 2a. The absorption spectrum shows a strong peak at $272 \mathrm{~nm}$ and broad bands at $300-400 \mathrm{~nm}$, indicating the results from carboxylation of $\mathrm{C}_{60}$-TEG which has weak broad band at $260 \mathrm{~nm}$ and $350 \mathrm{~nm}$ [14]. These features are similar to those of the spectra of the hydrophilic fullerene carboxylic acid derivative and fullerenol, respectively [23, 24]. In addition, the fluorescence spectrum of $\mathrm{C}_{60}$-TEG-COOH exhibits broad fluorescence with a maximum peak at $500 \mathrm{~nm}$ under excitation at $350 \mathrm{~nm}$, which is slightly

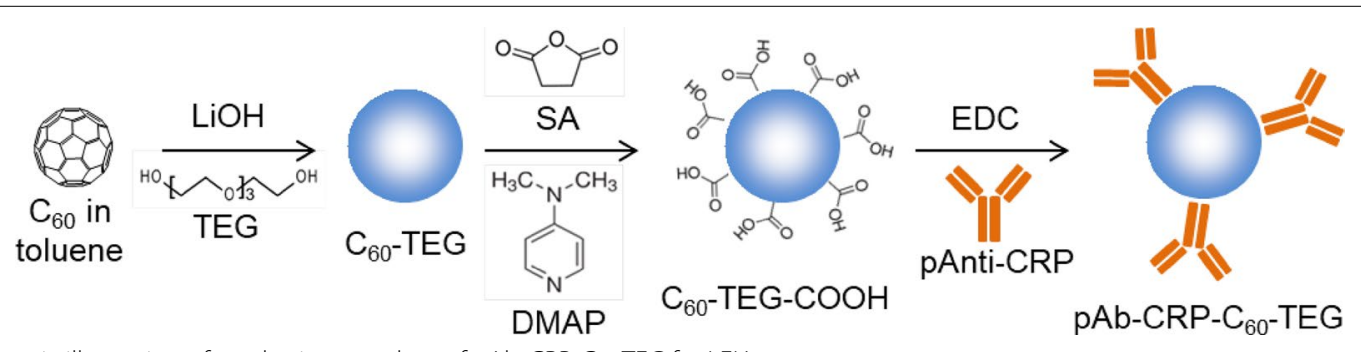

Fig. 1 Schematic illustration of synthetic procedure of pAb-CRP-C ${ }_{60}$-TEG for LFIA
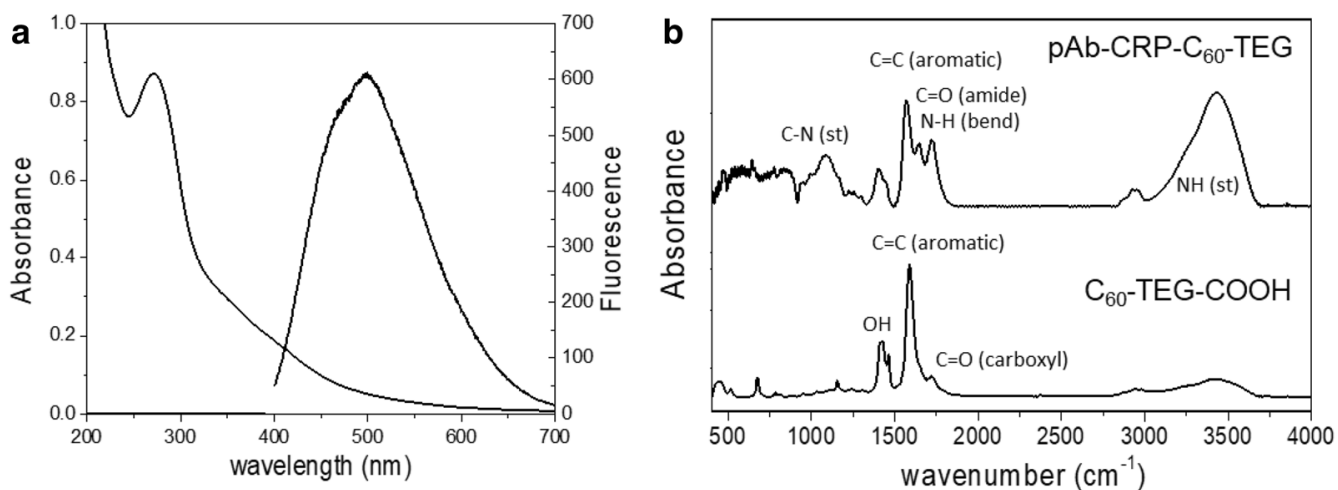

Fig. 2 a Absorption and fluorescence spectra of $\mathrm{C}_{60}-\mathrm{TEG}-\mathrm{COOH}$. b Infrared spectra of $\mathrm{C}_{60}$-TEG-COOH and pAb-CRP- $\mathrm{C}_{60}{ }^{-T E G}$ 
blue-shifted compared to the fluorescence of $\mathrm{C}_{60}$-TEG, meaning that the carboxylation may lead to change of the optical properties from $\mathrm{C}_{60}$-TEG. However, the fluorescence quantum efficiency of $\mathrm{C}_{60}$-TEG-COOH $\left(\Phi_{\mathrm{F}}=0.33\right)$ was higher than that of $\mathrm{C}_{60}$-TEG $\left(\Phi_{\mathrm{F}}=0.095\right)$ so that it is sufficient to use as a fluorescent probe for LFIA.

After conjugating pAb-CRP with $\mathrm{C}_{60}$-TEG-COOH via an EDC coupling reaction, we used infrared spectroscopy to examine the antibody conjugation with the $\mathrm{C}_{60}$-TEG-COOH. The infrared spectra in Fig. $2 \mathrm{~b}$ reveal that the $\mathrm{C}_{60}$-TEG-COOH was successfully conjugated with pAb-CRP by the formation of amide bond $(\mathrm{C}=\mathrm{O}$ amide at $1645 \mathrm{~cm}^{-1}$ and $\mathrm{N}-\mathrm{H}$ stretch at $3430 \mathrm{~cm}^{-1}$ ). $\mathrm{C}-\mathrm{N}$ stretch at $1081 \mathrm{~cm}^{-1}$ shows the presence of amine bond from the antibody. In comparison, the simple carboxylic acid peaks appeared at $1425 \mathrm{~cm}^{-1}$ (OH bending) and $1716 \mathrm{~cm}^{-1}(\mathrm{C}=\mathrm{O}$ stretching) in the spectrum of unconjugated $\mathrm{C}_{60}$-TEG-COOH. We also determined, via the Bradford assay, that the amount of conjugated pAb$\mathrm{CRP}$ was $4.58 \mu \mathrm{g} / \mathrm{mg}$ of $\mathrm{C}_{60}$-TEG-COOH.

\subsection{Preparation and optimization of $\mathrm{C}_{60}$-TEG-based LFIA}

By using the pAb-CRP- $\mathrm{C}_{60}$-TEG, we designed the fluorescent immunochromatographic assay on a lateral flow strip for the rapid and quantitative detection of CRP (Fig. 3). First, the pAb-CRP- $\mathrm{C}_{60}$-TEG was dispensed on the conjugate pad of a lateral flow test strip. The antimouse IgG and mAb-CRP were placed on the nitrocellulose membrane using a dispenser, forming the $\mathrm{CL}$ and $\mathrm{TL}$, respectively. For the detection of CRP, the sample solution was applied onto the sample pad. Then, the solution migrated to the conjugate pad, where the CRP can bound with pAb CRP-C 60 -TEG. Next, free pAb-CRP$\mathrm{C}_{60}$-TEG and CRP-binding pAb-CRP- $\mathrm{C}_{60}$-TEG were captured by anti-IgG in the $\mathrm{CL}$ and $\mathrm{mAb}-\mathrm{CRP}$ in the
TL, respectively. After 15 min of migration, the strip was subjected to a fluorescence measurement system for the determination of the TL/CL fluorescence signal ratio.

We optimized the type of blocking solution and its dilution factors to reduce the non-specific adsorption of pAb-CRP- $\mathrm{C}_{60}$-TEG during flow through the strip. After testing three different blocking solutions, each containing casein, skim milk, and BSA in up to tenfold dilutions, we found that $1 \%$ BSA solution was the most efficient in obtaining a fluorescence response with a high signalto-noise ratio. Moreover, other parameters, e.g., the volume of sample solution, the washing steps with PBS to remove the unbound fluorescent conjugates, and the immunoreaction time, were also optimized to increase the sensitivity.

\subsection{Quantitative detection of CRP using $\mathrm{C}_{60}$-TEG-based LFIA}

On the basis of the optimal experiment conditions, the analytical performance for quantitative measurement of CRP was further evaluated with standard CRP samples in serum. Figure 4a shows fluorescence images of the TLs and CLs on the test strip with various CRP concentrations as high as $10 \mathrm{ng} / \mathrm{ml}$. In the absence of CRP, the CL was easily observed, whereas the TL was not observed. Both the TL and the CL were visible in the presence of CRP, and the lines became more vivid as the CRP concentration was increased, indicating that additional pAbCRP- $\mathrm{C}_{60}-\mathrm{TEG}$ was captured on the TL. The fluorescence signal ratio of the TL and CL, as analyzed by image analysis software, is shown in Fig. 4b. The relative ratio of TL/ CL linearly increases with increasing CRP concentration in the range of $0.1-10 \mathrm{ng} / \mathrm{ml}$. These results are comparable with those of previous studies on the detection of CRP using various methods, including commercial- and

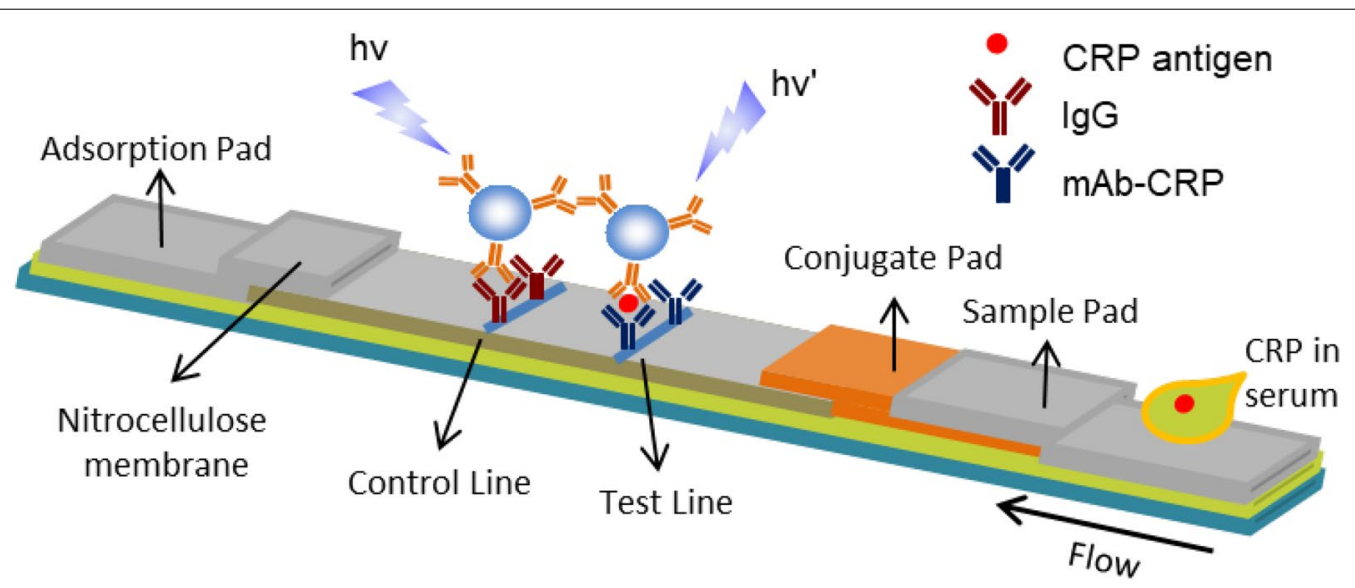

Fig. 3 Schematic diagram of $\mathrm{C}_{60}$-TEG-based LFIA for the detection of CRP 


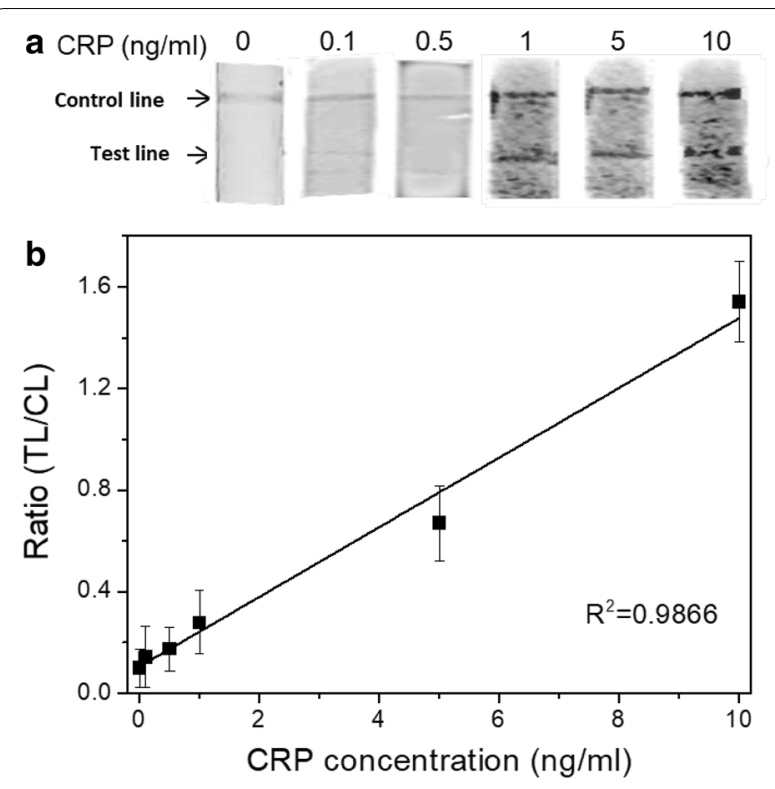

Fig. 4 a The fluorescence images of the test strips with various concentration of CRP from 0.01 to $10 \mathrm{ng} / \mathrm{ml}$. b The calibration curve obtained from the area ratio $(T L / C L)$ against the concentration of CRP in serum

microfluidic-based point-of-care immunodiagnostics [25, 26].

Immunochromatographic strips offer benefits because of their user-friendly format, short test times, long-term stability, and relatively low fabrication costs. Although CG-based strip sensors are a standard immunochromatographic method, this method suffers from limitations such as low sensitivity and color interference in hemolytic samples. However, the fluorescent LFIA provides advantages such as high sensitivity, quantitative measurement, and lack of color interference. In this study, fluorescent fullerene nanoparticles $\left(\mathrm{C}_{60}\right.$-TEG) were used as a new reporting material in a fluorescent LFIA. The $\mathrm{C}_{60}$-TEG can be simply prepared and modified to conjugate antibodies and is adaptable in a nitrocellulose strip because of its hydrophilicity and relatively small size, which may facilitate flow through the membrane.

\section{Conclusion}

A new fluorescent LFIA using $\mathrm{C}_{60}$-TEG was developed for the detection of a wide range of CRP concentrations. The $\mathrm{C}_{60}$-TEG-COOH was conjugated with $\mathrm{pAb}$ CRP via an EDC reaction, and the conjugates were used as a fluorescent probe as they migrated from the conjugate pad to the CL and TL, which were printed with anti-mouse IgG and $\mathrm{mAb}-\mathrm{CRP}$ in a strip, respectively. The TL/CL fluorescence signal ratio increased as the CRP concentration was increased from 0.1 to $10 \mathrm{ng} / \mathrm{ml}$ in serum. The fluorescent fullerene nanoparticle-based LFIA was simply prepared and successfully used to detect a wide range of CRP concentrations. Therefore, the combined fluorescent fullerene nanoparticle-based LFIA exhibits strong potential for highly sensitive, rapid, and quantitative immunoassays.

\section{Experimental section}

\subsection{Materials and chemicals}

Buckminsterfullerene $\left(\mathrm{C}_{60}, 99.9 \%\right)$ was purchased from SES Res. (TX, USA). Toluene (99.8\%), tetraethylene glycol (TEG), lithium hydroxide (LiOH), dimethyl sulfoxide (DMSO), succinic anhydride (SA), 4-(dimethylamino)pyridine (DMAP), diethyl ether (DE), EDC, anti-mouse immunoglobulin G (anti-mouse IgG), and bovine serum albumin (BSA) were purchased from Sigma-Aldrich (St. Louis, MO, USA). Ethyl acetate (EA, 99\%) was obtained from Daejung (Seoul, Korea). Ethanol was obtained from Merck (Darmstadt, Germany). Dulbecco's phosphate-buffered saline (PBS) was obtained from Gibco (NY, USA). CRP and polyclonal CRP antibody (pAb-CRP) were purchased from Abcam. Monoclonal CRP antibody (mAb-CRP) was obtained from $R \& D$ Systems. All chemicals were used without further purification.

\subsection{Synthesis and characterization of $\mathrm{C}_{60}$-TEG-COOH}

The fluorescent fullerene NPs were synthesized using a modified version of a process reported in the literature [14]. First, $\mathrm{C}_{60}$ solution ( $10 \mathrm{ml}$ in toluene) at a concentration of $0.25 \mathrm{mg} / \mathrm{ml}$ was added to $10 \mathrm{ml}$ of TEG. Next, $\mathrm{LiOH}\left(40 \mathrm{mg}\right.$ ) was added to the mixture of $\mathrm{C}_{60}$ and TEG, turning the color of solution from pink to dark-brown within $10 \mathrm{~min}$. After stirring of the solution for $20 \mathrm{~h}$, the resultant fullerene NPs were precipitated by the addition of excess EA. The precipitates were collected by filtration and dried to obtain $\mathrm{C}_{60}$-TEG powders. To conjugate the anti-CRP, the $\mathrm{C}_{60}$-TEG was firstly functionalized to expose carboxylic acid groups on the surface of the NPs. $10 \mathrm{mg}$ of $\mathrm{C}_{60}$-TEG powder was dissolved in $1 \mathrm{ml}$ of DMSO, and $50 \mathrm{mg}$ of SA and $15 \mathrm{mg}$ DMAP were added to the $\mathrm{C}_{60}$-TEG solution. After the solution was stirred for $20 \mathrm{~h}$, the resultant NPs were precipitated by the addition of excess DE. The precipitates were collected by filtration and dried to obtain carboxylated $\mathrm{C}_{60}$-TEG $\left(\mathrm{C}_{60}\right.$-TEG-COOH $)$ powder. The optical and chemical properties of $\mathrm{C}_{60}$-TEG-COOH were analyzed by $\mathrm{UV} /$ Visible spectroscopy (Beckman Coulter, DU-800, USA), fluorescence spectroscopy (Perkin-Elmer, LS55, UK), and FTIR spectrophotometry (Bruker Optics, IF66, USA) using the KBR-pellet method. 


\subsection{Preparation of $\mathrm{pAb}-\mathrm{CRP}-\mathrm{C}_{60}$-TEG}

The pAb-CRP- $\mathrm{C}_{60}$-TEG was prepared by an EDC coupling reaction between $\mathrm{C}_{60}-\mathrm{TEG}-\mathrm{COOH}$ and $\mathrm{pAb}-\mathrm{CRP}$. The $\mathrm{C}_{60}$-TEG-COOH was dissolved in PBS at a concentration of $10 \mathrm{mg} / \mathrm{ml}$, and $10 \mu \mathrm{l}$ of $0.1 \mathrm{M} \mathrm{EDC}$ and 10 $\mu \mathrm{l}$ of $0.1 \mathrm{mg} / \mathrm{ml} \mathrm{pAb-CRP} \mathrm{were} \mathrm{added} \mathrm{to} \mathrm{the} 100 \mu \mathrm{l}$ of $\mathrm{C}_{60}$-TEG-COOH solution. The reaction mixture was maintained at room temperature for $1 \mathrm{~h}$. The resulting mixture (pAb-CRP- $\mathrm{C}_{60}$-TEG) was purified by spin chromatography using a PD Spin Trap G-25 column (GE Healthcare) and collected as eluates after centrifugation of the column at $2500 \mathrm{rpm}$ for $2 \mathrm{~min}$. Finally, 1\% BSA $(\mathrm{w} / \mathrm{v})$ solution was further added to the eluted solution for blocking.

\subsection{Preparation of a lateral flow immunochromatographic test strip}

The test strip involved (i) a sample pad, (ii) a nitrocellulose (NC) membrane, and (iii) an absorption pad, and all attached to a backing card. The test zone involved immobilized $\mathrm{mAb}$-CRP $(0.5 \mathrm{mg} / \mathrm{ml}$ in PBS) as the test line (TL) and anti-mouse IgG (1 mg/ml in PBS) as the control line $(\mathrm{CL})$, both of which were dispensed at $1 \mu \mathrm{l} / \mathrm{cm}$ using an automatic dispenser. After drying for $1 \mathrm{~h}$ at $37^{\circ} \mathrm{C}$ in an incubator, the membranes were reacted with a blocking solution $\left(1 \mathrm{mg} / \mathrm{ml} \mathrm{BSA}\right.$ in PBS) for $30 \mathrm{~min}$ at $20^{\circ} \mathrm{C}$. After the absorption pad and the sample pad were affixed to the top and the bottom of the membrane, respectively, the membranes were cut into strips 3-4 $\mathrm{mm}$ wide using an automatic programmable cutter (GCI-800, Guillotine Cutting, ZETA Corporation, Korea). The diluted pAb-CRP- C $_{60}$-TEG was dispensed onto the conjugating pad prior to the plate being pasted on with an overlap of $2 \mathrm{~mm}$ with the NC membrane. The sample pad was pasted onto the same end with its margin justified to the conjugating pad. Finally, after being sealed, the test strip was stored in a desiccator until use.

\subsection{Detection of CRP using $\mathrm{C}_{60}$-TEG-based LFIA}

Various concentrations of CRP solution were prepared in human serum solution. The LFIA strips were dipped into 96-well plate wells that contained CRP solution $(0.1 \mathrm{ml})$. After $15 \mathrm{~min}$, the fluorescence signals of both the CL and the TL on the strips were measured using a luminescence image analyzer (LAS-3000, FujiFilm) and excitation and emission wavelengths of 460 and $510 \mathrm{~nm}$, respectively. The fluorescence intensities of the TL and CL were analyzed from a camera image using the MultiGauge 3.0 software.

\section{Acknowledgements}

This research was supported by the Basic Science Research Program through the National Research Foundation of Korea (NRF) funded by the
Ministry of Science and ICT (MSIT) (NRF-2019R1C1C1006084 and NRF2019R1 C1 C1006867), the Center for BioNano Health-Guard funded by the MSIT of Korea as Global Frontier Project (H-GUARD_2013M3A6B2078950), the Bio and Medical Technology Development Program of the NRF funded by MSIT of Korea (NRF-2018M3A9E2022821), and KRIBB initiative Research Program.

\section{Authors' contributions}

KMP takes the lead through the whole experiments. DJC and MC performed the synthesis and characterization of NPs. KMP, DJC and MC performed the detection of CRP by using $\mathrm{C}_{60}$-TEG-based LFIA. JJ conceived of the idea and supervised the project. TK and JJ mainly wrote the manuscript and all authors provided feedback and helped the completion of the final manuscript. All authors read and approved the final manuscript.

\section{Funding}

This research was supported by the Basic Science Research Program through the National Research Foundation of Korea (NRF) funded by the Ministry of Science and ICT (MSIT) (NRF-2019R1C1C1006084 and NRF2019R1C1C1006867), the Center for BioNano Health-Guard funded by the MSIT of Korea as Global Frontier Project (H-GUARD_2013M3A6B2078950), the Bio and Medical Technology Development Program of the NRF funded by MSIT of Korea (NRF-2018M3A9E2022821), and KRIBB Research Initiative Program.

\section{Availability of data and materials}

All data generated or analysed during this study are included in this published article.

\section{Competing interests}

The authors declare that they have no competing interests.

\section{Author details}

${ }^{1}$ BioNano Health Guard Research Center, Korea Research Institute of Bioscience and Biotechnology, Daejeon 34141, Republic of Korea. ${ }^{2}$ Bionanotechnology Research Center, Korea Research Institute of Bioscience and Biotechnology, Daejeon 34141, Republic of Korea. ${ }^{3}$ Department of Nanobiotechnology, KRIBB School of Biotechnology, University of Science \& Technology,

Daejeon 34113, Republic of Korea. ${ }^{4}$ Environmental Disease Research Center, Korea Research Institute of Bioscience and Biotechnology, Daejeon 34141, Republic of Korea.

Received: 27 July 2019 Accepted: 14 October 2019

Published online: 01 November 2019

\section{References}

1. C. Chen, J. Wu, Sensors 12, 11684 (2012)

2. R. Tanaka, T. Yuhi, N. Nagatani, T. Endo, K. Kerman, Y.Takamura, E. Tamiya, Anal. Bioanal. Chem. 385, 1414 (2006)

3. Y. Xing, P. Wang, Y. Zang, Y. Ge, Q. Jin, J. Zhao, X. Xu, G. Zhao, H. Mao, Analyst 138, 3457 (2013)

4. S.G. Hwang, K. Ha, K. Guk, D.K. Lee, G. Eom, S. Song, T. Kang, H. Park, J. Jung, E.-K. Lim, Sci. Rep. 8, 12999 (2018)

5. T.-H. Ulep, J.-Y. Yoon, Nano Converg. 5, 14 (2018)

6. J. Hu, S. Wang, L. Wang, F. Li, B. Pingguan-Murphy, T.J. Lu, F. Xu, Biosens. Bioelectron. 54, 585 (2014)

7. Z. Li, Y. Wang, J. Wang, Z. Tang, J.G. Pounds, Y. Lin, Anal. Chem. 82, 7008 (2010)

8. R.S. Niedbala, H. Feindt, K. Kardos, T. Vail, J. Burton, B. Bielska, S. Li, D. Milunic, P. Bourdelle, R. Vallejo, Anal. Biochem. 293, 22 (2001)

9. J.-M. Park, H.-W. Jung, Y.W. Chang, H.-S. Kim, M.-J. Kang, J.-C. Pyun, Anal. Chim. Acta 853, 360 (2015)

10. C. Suárez-Pantaleón, J. Wichers, A. Abad-Somovilla, A. van Amerongen, A. Abad-Fuentes, Biosens. Bioelectron. 42, 170 (2013)

11. Q.-Y. Xie, Y.-H. Wu, Q.-R. Xiong, H.-Y. Xu, Y.-H. Xiong, K. Liu, Y. Jin, W.-H. Lai, Biosens. Bioelectron. 54, 262 (2014)

12. Q. Yang, X. Gong, T. Song, J. Yang, S. Zhu, Y. Li, Y. Cui, Y. Li, B. Zhang, J. Chang, Biosens. Bioelectron. 30, 145 (2011) 
13. F. Zhang, M. Zou, Y. Chen, J. Li, Y. Wang, X. Qi, Q. Xue, Biosens. Bioelectron. 51, $29(2014)$

14. J. Jeong, J. Jung, M. Choi, J.W. Kim, S.J. Chung, S. Lim, H. Lee, B.H. Chung, Adv. Mater. 24, 1999 (2012)

15. A.S. Stasheuski, V.A. Galievsky, A.P. Stupak, B.M. Dzhagarov, M.J. Choi, B.H. Chung, J. Jeong, Photochem. Photobiol. 90, 997 (2014)

16. P.H. Mackie, R.A. Crockson, J. Stuart, Q.E. Hospital, J. Clin. Pathol. 32, 1253 (1979)

17. P.M. Ridker, M. Cushman, M.J. Stampfer, R.P.Tracy, C.H. Hennekens, Circulation 97, 425 (1998)

18. A. Hwang, E. Kim, J. Moon, H. Lee, M. Lee, J. Jeong, E.-K. Lim, J. Jung, T. Kang, B. Kim, A.C.S. Appl, Mater. Interfaces 11, 18960 (2019)

19. J.S. Ahn, S. Choi, S.H. Jang, H.J. Chang, J.H. Kim, K.B. Nahm, S.W. Oh, E.Y Choi, Clin. Chim. Acta 332, 51 (2003)

20. W. Leung, C.P. Chan, T.H. Rainer, M. Ip, G.W.H. Cautherley, R. Renneberg, J. Immunol. Methods 336, 30 (2008)
21. S.W. Oh, J.D. Moon, S.Y. Park, H.J. Jang, J.H. Kim, K.B. Nahm, E.Y. Choi, Clin. Chim. Acta 356, 172 (2005)

22. C. Swanson, A. D'Andrea, Clin. Chem. 59, 641 (2013)

23. J.D. Driskell, C.A. Jones, S.M. Tompkins, R.A. Tripp, Analyst 136, 3083 (2011)

24. V.K. Periya, I. Koike, Y. Kitamura, S.I. Iwamatsu, S. Murata, Tetrahedron Lett. 45, 8311 (2004)

25. N. Brouwer, J. van Pelt, Clin. Chim. Acta 439, 195 (2015)

26. L. Gervais, E. Delamarche, Lab Chip 9, 3330 (2009)

\section{Publisher's Note}

Springer Nature remains neutral with regard to jurisdictional claims in published maps and institutional affiliations.

\section{Submit your manuscript to a SpringerOpen ${ }^{\circ}$ journal and benefit from:}

- Convenient online submission

- Rigorous peer review

- Open access: articles freely available online

- High visibility within the field

- Retaining the copyright to your article

Submit your next manuscript at $\boldsymbol{\nabla}$ springeropen.com 